\title{
Review Article \\ Research Progress on Mechanism of Podocyte Depletion in Diabetic Nephropathy
}

\author{
Haoran Dai, ${ }^{1}$ Qingquan Liu, ${ }^{1,2}$ and Baoli Liu ${ }^{1,2}$ \\ ${ }^{1}$ Department of Nephrology, Shunyi Branch, Beijing Hospital of Traditional Chinese Medicine, Station East 5, Shunyi District, \\ Beijing 101300, China \\ ${ }^{2}$ Beijing Hospital of Traditional Chinese Medicine Affiliated to Capital Medical University, 23 Meishuguanhou Street, \\ Dongcheng District, Beijing 100010, China
}

Correspondence should be addressed to Qingquan Liu; liuqingquan2003@126.com and Baoli Liu; polyliu2000@sina.com

Received 23 December 2016; Revised 5 February 2017; Accepted 5 March 2017; Published 16 July 2017

Academic Editor: Wei J. Liu

Copyright (C) 2017 Haoran Dai et al. This is an open access article distributed under the Creative Commons Attribution License, which permits unrestricted use, distribution, and reproduction in any medium, provided the original work is properly cited.

\begin{abstract}
Diabetic nephropathy (DN) together with glomerular hyperfiltration has been implicated in the development of diabetic microangiopathy in the initial stage of diabetic diseases. Increased amounts of urinary protein in DN may be associated with functional and morphological alterations of podocyte, mainly including podocyte hypertrophy, epithelial-mesenchymal transdifferentiation (EMT), podocyte detachment, and podocyte apoptosis. Accumulating studies have revealed that disruption in multiple renal signaling pathways had been critical in the progression of these pathological damages, such as adenosine monophosphate-activated kinase signaling pathways (AMPK), wnt/ $\beta$-catenin signaling pathways, endoplasmic reticulum stressrelated signaling pathways, mammalian target of rapamycin (mTOR)/autophagy pathway, and Rho GTPases. In this review, we highlight new molecular insights underlying podocyte injury in the progression of DN, which offer new therapeutic targets to develop important renoprotective treatments for DN over the next decade.
\end{abstract}

\section{Introduction}

Diabetic nephropathy (DN), as the primary cause of endstage renal failure, is one of the most serious complications in diabetic patients, which develops in up to $30 \%-$ $40 \%$ of patients with types 1 or 2 diabetes mellitus [1]. An early sign of $\mathrm{DN}$ is an increased amount of urinary protein and characterized by mesangial nodular hyperplasia and thickening of the glomerular basement membrane (GBM). Microalbuminuria plays an important role in the change of the GBM. Additionally, a detailed renal biopsy data analysis regarding diabetics showed that diabetic kidney damage would include visceral epithelial cells and sertoli cell dysfunction [2, 3]. Although some researches demonstrated that podocyte injury had association with the development of DN [4], the mechanisms underlying this association are still not entirely understood and need to be further investigated.

\section{Proteinuria and Podocyte Injury in DN}

Glomerular epithelial cells, also called podocytes, are highly specialized cells, which are composed of cytoskeletal structure, joint connection, and branching foot processes circling the GBM. Podocytes, as terminal differentiation cells, are important functional cells in the glomerulus and can not regenerate when they suffer from injury. Their damage and apoptosis could result in the destruction of the glomerular filtration membrane and induce unfavorable factors in DN [5]. Foot processes are consisted of basal aspects, basolateral region and parietal region, which firmly adhere to the GBM through podoplanin protein. The basolateral region was found interdigitating with the neighboring basolateral region by slit diaphragm (SD) [6]. Furthermore, transmembrane proteins connect adapter proteins with actin cytoskeleton to maintain filtration barrier structure and function. In the podocyte parietal region, salivary proteins are used to 
maintain a filtration way among adjacent foot processes in the GBM through sufficient negatively charging [7]. Podocytes play an important role in pathological mechanisms underlying DN. Imasawa et al. [8] declared that structure and function in podocyte molecules transformed in highglucose conditions, which resulted from suppression of myocyte-specific enhancer factor $2 \mathrm{C}(\mathrm{MEF} 2 \mathrm{C})$ and myogenic factor 5 (MYF5) expressions through using a conditionally differentiating human podocyte cell line.

\section{Pathomechanism of Podocyte Injury}

3.1. Podocyte Hypertrophy. Although the pathophysiology of podocyte hypertrophy in the initial stage of $\mathrm{DN}$ is still ambiguous, animal and human studies have established that glomerular podocyte hypertrophy was associated with the development of DN $[9,10]$. Previous researches indicated that MAPK, TGF- $\beta$, and AngII had different effects on mesangial matrices and cells, leading to glomerular hypertrophy in the progress of DN. However, elaborate mechanisms underlying podocyte hypertrophy were less reported [11]. Romero et al. [12] concluded that AngII increased expressions of parathyroid hormone-related protein (PTHrP), TGF- $\beta 1$, and cell cycle regulatory protein-p27Kip, which promoted the aggravation of podocyte hypertrophy in high glucose. The mammalian target of rapamycin (mTOR) signaling mainly consisted of mTORC1 and mTORC2. Several studies suggested that mTORC1 was closely associated with the activation of podocyte hypertrophy which was induced by high glucose [13]. In the early stage of diabetes, it was obviously found that high filtration of glomerular is accompanied with podocyte hypertrophy. In general, mature podocytes had to expand the size of themselves in order to compensate for glomerular dilation, which contributed to cover the denuded region of the GBM, because they were terminal-differentiated cells, which no longer are proliferated $[14,15]$. Jo et al. [16] declared that interleukin 6 (IL-6) and its downstream cascade signaling proteins, such as Gp130 signal transducer and activator of transcription 3 (STAT3) signal transducer, were key regulators related to podocyte hypertrophy in a high-glucose environment. In general, hyperglycemia induced the overexpression of nuclear STAT3 via the activation of upstream signal transduction element Gp130, which is eventually leading to podocyte hypertrophy. Kim et al. [17] found that TCTP, as a mediating signal of cell growth, was overexpressed with high percentage in the glomeruli of diabetic mice and gave rise to podocyte hypertrophy. Fluorescent double-labeling method indicated that TCTP was mainly observed in podocytes. Studies showed that TCTP could activate the mTORC1 signal pathway and promote high expression of CKIs, which caused podocyte cycle arrest and hypertrophy. On the contrary, overexpression of mTORC1 and CKIs could be inhibited by gene knockout of TCTP, to make the podocyte bodies smaller. Meanwhile, in vitro experiments indicated that TCTP inhibitor could downregulate the expression of CKIs, ameliorating podocyte hypertrophy caused by high glucose. Kim et al. [18] showed that AngII could also upregulate protein expressions of kinases ERK1/2 and Akt/PKB, which contributed to podocyte hypertrophy. Hence, it has been shown that all ERK1/2, Akt/PKB, IL-6/JAK2/STAT3, and mTOR signalpathway activities had important roles in podocyte hypertrophy (Figure 1).

3.2. Podocyte Epithelial-Mesenchymal Transition. Previous studies have shown a connection between podocyte apoptosis and proteinuria. However, more and more studies have demonstrated that normal epithelial cells showed phenotype conversion of a variety of nephropathies [19]. Cells lost their original features when the pathological process of EMT occurred, which induced disappeared cell contact, damaged cell polarity, and recaptured characteristics of the mesenchymal markers, such as vimentin, $\alpha$-smooth muscle actin $(\alpha$-SMA), and fibroblast-specific protein 1 (FSP1). FSP1 is one of the important members of calcium-binding protein S100 family and also a fibroblast-specific protein without epithelial cells [20]. Many studies showed that renal tubular epithelial cells and podocytes were activated after acute (48$72 \mathrm{~h}$ ) exposure of cells to elevated glucose levels or other stimulations of diabetes, which resulted in less protein expressions of E-cadherin and ZO-1. Conversely, expressions of transdifferentiation proteins, such as $\alpha$-SMA and vimentin, were immediately increased after these stimulation [21-23]. In the initial stage of STZ-induced diabetes, morphology of podocytes were damaged, accompanied with increased expression of the podocyte marker, nephrin protein, and a fall in the mesenchymal marker, desmin protein [24]. Yamaguchi et al. [25] found that FSP1-positive cells were significantly increased in urinary sediment and approximately attached to 86 percent of total podocytes in 109 type 2 diabetes patients. 43 of these patients with massive proteinuria in this study experienced kidney biopsy. The FSP1 positive cells selectively expressed Snail and ILK preferentially, which played pivotal roles in inducing EMT. Xing et al. [26] demonstrated that podocyte incubated in elevated glucose levels for $48 \mathrm{~h}$ could trigger activation of the PI3K/AKT pathway and elevate protein expressions of $\alpha$-SMA and desmin. Whereas, protein expressions of podocalyxin and nephrin were suppressed. Functionally, it is apparently speculated that podocyte EMT may be tightly related to the PI3K/AKT signal pathway. Li et al. [27] found that the elevated glucose level upregulated protein expression of Snail and suppressed protein expressions of P-cadherin and nephrin in vitro. The change above decreased podocyte-related proteins of nephrin and ZO-1, elevated expressions of desmin, MMP9, and FSP1 in sequence. As shown in Figure 2, podocyte EMT widely participated in the early stage of podocyte deletion in diabetes mellitus via leading to podocyte detachment or podocyte apoptosis [28].

3.3. Podocyte Detachment. Podocytes and the glomerular basement membrane (GBM) are closely connected and then prevent the excretion of proteinuria via sustaining the glomerular filtration barrier. Researches showed that not only dead podocytes but also normal podocytes were found in the urinary sediment of patients with kidney disease. Furthermore, it had been concluded that podocytes could be cultured from urine of a healthy person [22]. One study 


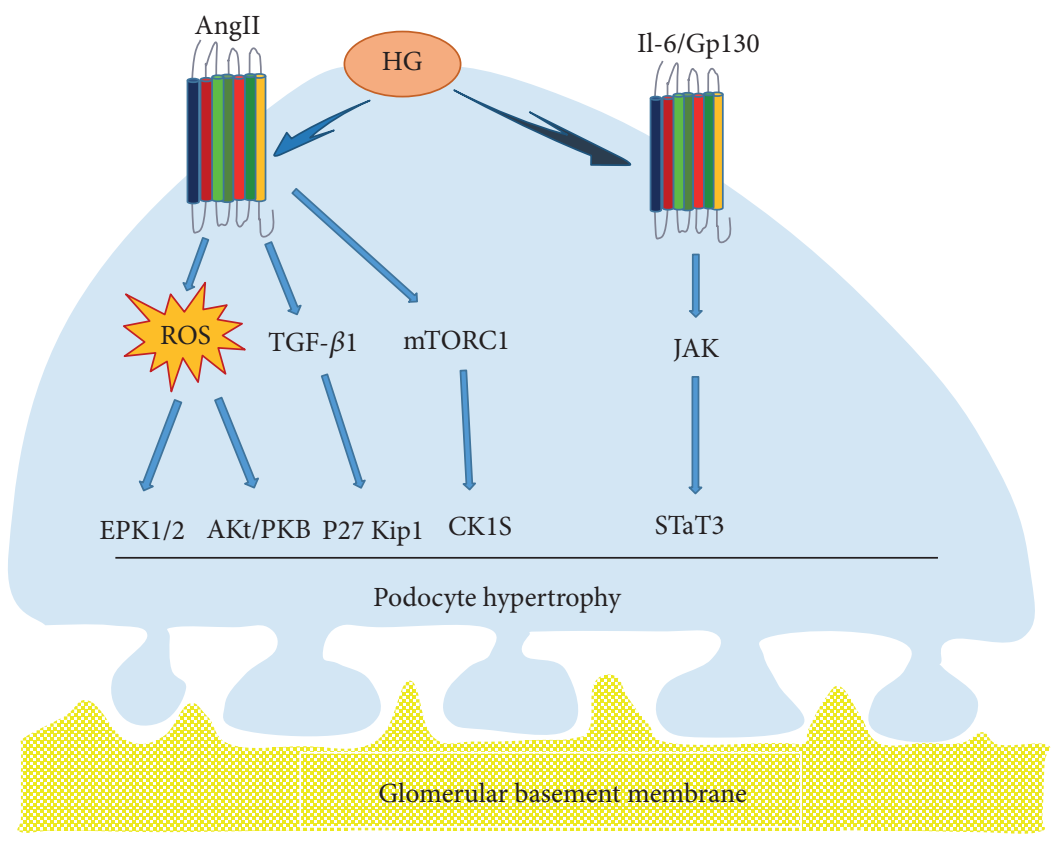

HG High glucose

FIGURE 1: Podocyte hypertrophy. In elevated glucose levels, AngII could elevate protein expressions of kinases ERK1/2 and Akt/PKB through ROS, trigger activation of p27Kip1 via TGF- $\beta 1$ signal pathway, or upregulate protein expression of CKIs by activating mTORC1, which eventually resulted in glomerular podocyte hypertrophy. Additionally, high glucose also induced podocyte hypertrophy via activation of the IL-6/Gp130-JAK/STAT3 signal pathway.

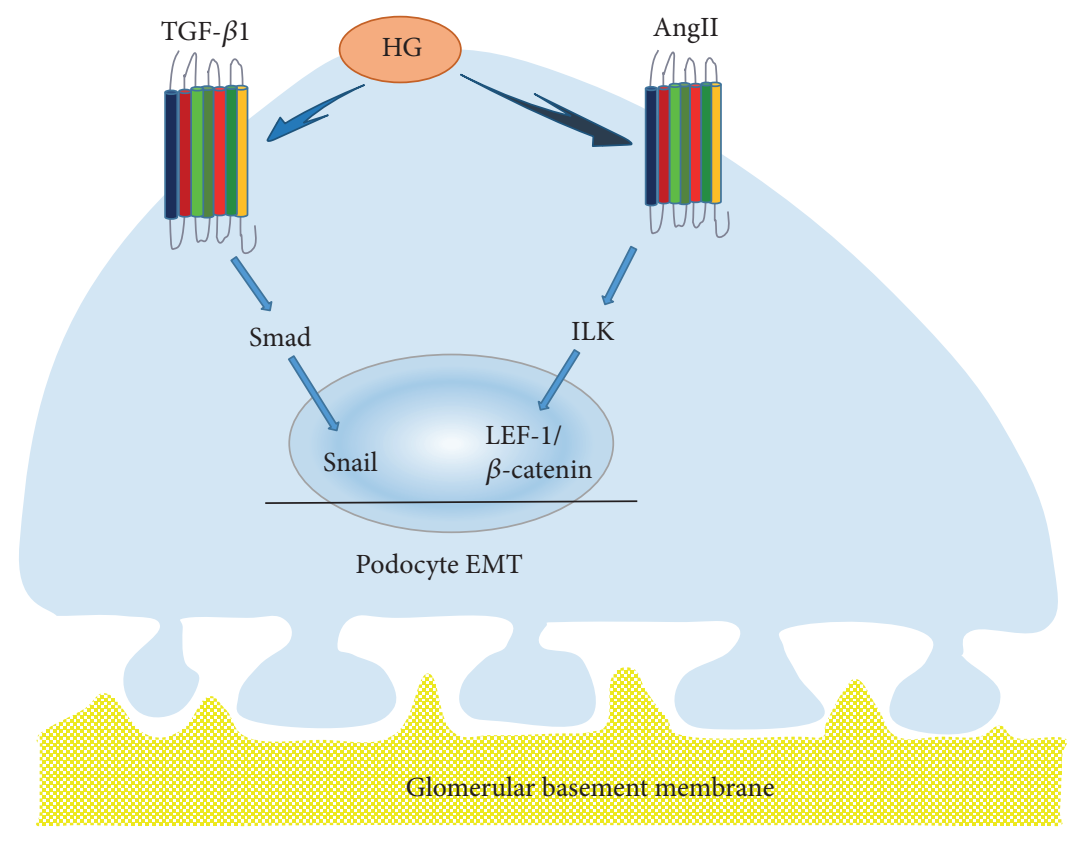

HG High glucose

FIGURE 2: Podocyte EMT. In elevated glucose levels, the TGF- $\beta 1 /$ Smad signal pathway resulted in increased protein expression of Snail in cultured podocytes, which induced podocyte EMT. Additionally, AngII promoted translocation of $\beta$-catenin/LEF-1 complexes into the nucleus through the enhancement of ILK in transitioning epithelia, where they downregulated CD2AP expression via promoting EMT transcription in podocytes. 


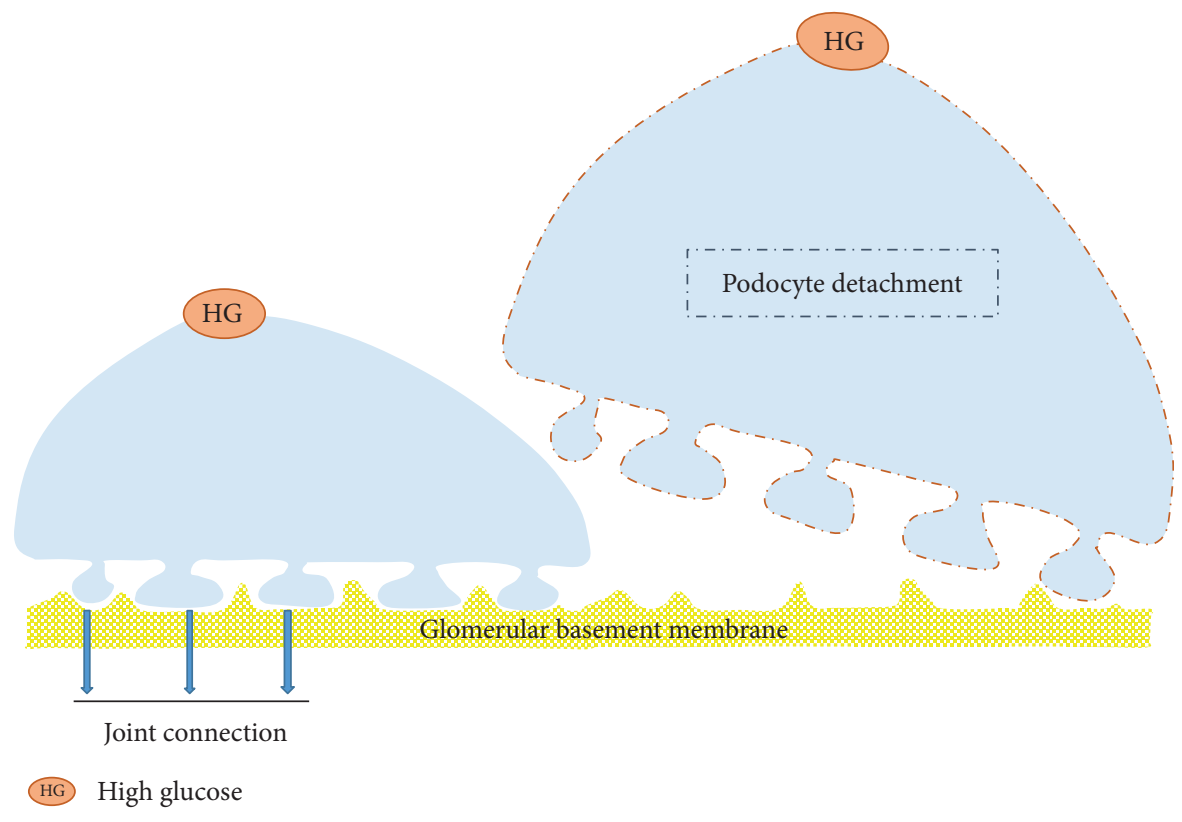

Figure 3: Podocyte detachment. Some podocytes became hypertrophic and stripped from the basement membrane in sequence, while the others tightly combined with basement membrane at the same stage of DN, which seems to be more related to podocyte EMT.

displayed that urinary podocyte might be used as an earlier biomarker of $\mathrm{DN}$ than proteinuria albuminuria in respect of renal injury [29]. It was generally known that integrin $\alpha 3 \beta 1$ was an important receptor which could tightly connect podocyte with the GBM [30]. Jim et al. [31] drew a conclusion that the expressions of podocyte marker proteins in the diabetic kidney, such as synaptopodin, podocin, and nephrin, were significantly decreased, which could result in podocyte cytoskeleton disorder, damaged sufficient adhesion, and separation between podocyte and the basement membrane. Hyperglycemia could downregulate expression of integrin $\alpha 3 \beta 1$ in both human and rat, as well as trigger activation of integrin-linked kinase (ILK). In addition, recent researches suggested that $\alpha 3 \beta 1$ participated in the adhesion function of podocyte $[32,33]$. Experimental researches on animals displayed that podocytes could break away from the glomerulus basement membrane in an artificial diabetic rat induced by streptozotocin (STZ) [34]. It was also suggested that both podocyte detachment and podocyte early changed in DN. However, whether podocyte detachment or podocyte hypertrophy appeared earlier was still difficult to distinguish [35]. It was obviously found that podocyte loss contributed to the development of DN. In the progression of disease, we found that in the same stage of DN, some podocytes became hypertrophy and detached from the basement membrane. Whereas, the others tightly combined with the basement membrane [36] (Figure 3).

3.4. Podocyte Apoptosis. Apoptosis pathway is involved in cellular growth and differentiation in many diseases, such as DN and IgA nephropathy. There are some evidences that podocyte apoptosis played a role in reduction in density and number of glomerular. Susztak et al. [37] found that mitochondria could activate nicotinamide adenine dinucleotide phosphate (NADPH) oxidase and reactive oxygen species (ROS) in high glucose, improve the expressions of p38 protein kinase and caspase 3 at the same time, then led to podocyte apoptosis, and produced much proteinuria. Glycosylation end products activated transcription factor FOXO4, which also induced podocyte apoptosis via p38 protein kinase signaling pathways [38]. In addition, the cytochrome P450 family raised hydroxyl and reduced coenzyme II twenty-four carbon olefine acid oxidase in high glucose, which increased the active oxygen class produces and prompting podocyte apoptosis [39]. Notch1 signal-dependent activation of p53 is a new podocyte apoptosis pathway [40]. A recent study found that podocyte apoptosis was closely related to the expression level of Notch, which then induced proteinuria and glomerular sclerosis. The activation of Notch signaling pathway may be included in DN all-acquired common mechanism of kidney disease [41]. Experiment with STZ-induced diabetes mouse displayed that the expressions of Jag, Notch, and ICN1 were increased immediately and the downstream component, such as Hesl and Heyl, were activated. It is also shown that the expressions of Bcl-2 and p53 were provoked, and the process eventually induced podocyte apoptosis [42]. Under normal circumstances, apoptosis-promoting and antiapoptosis signaling pathways coexisted in a same condition which maintained dynamic balance and guaranteed the stability environment. In general, phosphatidylinositol-3 kinase/ protein kinase in podocyte plays a crucial role in inhibition of podocyte apoptosis signaling pathways. The experiment also suggested that protein kinase phosphorylation reduced in $\mathrm{db} / \mathrm{db}$ mice, which may be an important inducement for podocyte apoptosis [43]. Liu et al. [44] found that podocyte apoptosis was associated with the disorder of cytoskeleton. Nestin is a VI intermediate filament protein related cell cytoskeleton which expressed in podocyte. The protein expression 


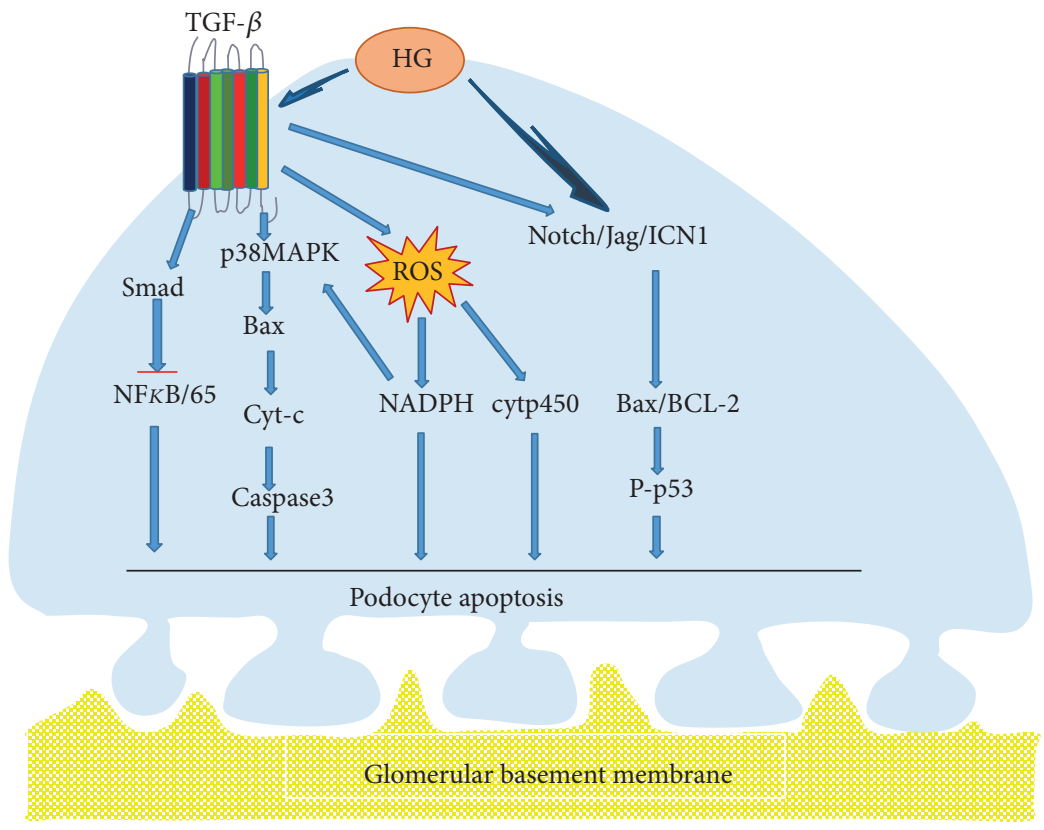

HG High glucose

FIGURE 4: Podocyte detachment. In elevated glucose levels, TGF- $\beta$ provoked p38 MAPK, Smad signal pathway and NADPH, and cytp450 to increase the protein expressions of Bax, cytochrome $\mathrm{C}$, and caspase-3, which resulted from overproduction of ROS. All of the above processes could result in podocyte apoptosis. They also stimulated the Notch/Jag/ICN1 signal pathway, which activated the Bcl-2 and p53 apoptotic pathways and induced podocyte apoptosis.

of nestin reduced in high glucose, however, podocyte apoptosis rate increased. TGF- $\beta 1$ could directly activate Smad7, which inhibit the activity of NF-kB and resulted in podocyte apoptosis. It also could provoke p38 MAP kinase, enhance the protein expressions of Bax and produce cytochrome $\mathrm{C}$, which activated caspase- 3 apoptosis pathway in sequence [45]. Liu et al. [46] demonstrated that metadherin was a potent modulator of podocyte apoptosis, which represented the target of miR-30 miRNAs, facilitating podocyte apoptosis via activating the HG-induced p38 MAPK-dependent pathway. Yao et al. [47] concluded that AS-IV inhibited podocyte apoptosis induced by high glucose, which reduced the expressions of TRPC6 and impaired the crosstalk of intracellular $\mathrm{Ca} 2+$ in podocytes. More and more evidences [48] indicated that AS-IV could protect the kidney from DN, including reduced podocyte damages and suppressed podocyte apoptosis through antioxidative stress and anti-inflammatory signaling pathways (Figure 4).

\section{Main Signaling Pathways of Podocyte Injury Mechanism in DN}

Many studies [49] suggested that DN podocyte injury was induced by the association of multiple factors, including mechanical stress, inflammatory reaction, oxidative stress, TGF- $\beta 1$ induction, renin angiotensin aldosterone system (RAAS) activation, and AGEs accumulation. And there are lots of signaling pathways involved in the regulation mTOR signaling pathways mediated by autophagy, adenosine monophosphate-activated kinase (AMPK) signaling pathway, Wnt/ $\beta$-catenin signaling pathway, and so on.

4.1. Adenosine Monophosphate-Activated Kinase Signaling Pathways (AMPK) in DN. AMPK is not only a serine protein kinase [50] playing a vital role in cells and tissues metabolisms of the diabetes progression but also one of the important metabolic emergency protein kinases. AMPK pathway is an autophagy-related signaling pathway composed of subunit heterologous proteins $\alpha, \beta$, and $\gamma$, which was activated as lack of energy in cells [51]. In the activation process, it could combine calmodulin-dependent kinase $\beta$ (CaMKK) and transform-activated kinase 1 (TAK-1). Then, it also effectively mediated intracellular calcium concentration and triggered the activation of the AMPK pathway to induce autophagy [52]. Conversely, AMPK could inhibit mTORCl activity and induce autophagy through TSCl/2-Rheb signaling pathways and/or phosphorus acidification of raptorrelated regulatory protein [53]. Meanwhile, AMPK also directly launch the phosphorylation of Ulkl/2 and induction of autophagy [54]. In addition, Sharma et al. [55] reported that adiponectin attenuated the induction of oxidative stress, reduced the synthesis of NADPH in podocyte, and simultaneously reduced albuminuria excretion in adiponectin-knockout mouse which could activate the AMPK pathway.

4.2. Wnt/ $\beta$-Catenin Signaling Pathways in DN. Wnt protein is one kind of secreted glycoprotein, containing a signal peptide and 23 or 24 conserved cysteine residues. It was activated 
via binding to ligand proteins and the Frizzled protein family. Wnts triggered a cascade of downstream reaction protein including axin, Disheveled, adenomatous polyposis coli (APC), and glycogen synthase kinase- (GSK-) $3 \beta$, which gave rise to phosphorylation of $\beta$-catenin in nuclei [56]. Researches showed that Wnt signaling pathway had effects on the differentiation, hyperplasia, maturation, and viability of cells [57]. It had been declared to be induced in DN, which played a crucial role in apoptosis and EMT formation of mesangial cells, podocyte, and tubular cells [58]. However, Dickkopf-related protein 1 (DKK1) is a secreted protein consisted of two cysteine abundant regions, which could reduce podocyte injury, decrease albuminuria, and protect the kidney by specific blocking $\mathrm{Wnt} / \beta$-catenin signal pathways $[59,60]$. Liu et al. [61] found that curcumin could prevent glomerular podocyte injury by inhibiting activated Wnt family members and $\beta$-catenin downstream effectors in obesityrelated glomerular disease model. Zhang et al. [62, 63] demonstrated that ubiquitin carboxy-terminal hydrolase-1 (UCH-L1) is abnormally expressed in injury podocytes, especially in immune-mediated disease. They also proved that the $\mathrm{Wnt} / \beta$-catenin signal pathway is promptly activated in podocyte, coinciding with overexpression of UCH-L1 induced by high glucose meanwhile [64]. Li et al. [65] announced that podocyte incubated in high glucose underwent injury, which attributed to the upregulation of transient receptor potential cation channel 6 (TRPC6) protein triggered by the classic $\mathrm{Wnt} / \beta$-catenin pathway.

\subsection{Endoplasmic Reticulum Stress-Related Signaling}

Pathways in DN. Recent studies suggested that endoplasmic reticulum stress was closely relevant to the injury of podocytes, endothelial cells, and mesangial cells in DN. It could induce glomerular obstacle of podocyte structure and function, participate in a variety of kidney diseases, and also lead to glomerular sclerosis [66]. Continuous endoplasmic reticulum stress had effects on the function of endoplasmic reticulum and could launch apoptosis signaling pathways which were mediated by endoplasmic reticulum stress at the same time and then activated the downstream apoptotic signaling molecules [67]. In patients with diabetes, hyperglycemia can motivate endoplasmic reticulum stress through a variety of ways, then cause cellular damage [68]. As important factors of $\mathrm{DN}$, advanced glycation end products (AGE) could upregulate the protein expressions of glucoseregulated protein 78 (GRP78) and induce endoplasmic reticulum stress depending on its dosage and time, eventually inducing apoptosis of podocyte [69]. In addition, high glucose and free fatty acids could induce endoplasmic reticulum stress as well as the occurrence of apoptosis in podocyte of rats, which could be inhibited via exogenous endoplasmic reticulum molecular chaperone [70]. Endoplasmic reticulum stress may aggravate podocyte dysfunction in the early stage of DN [71]. The relationship between endoplasmic reticulum stress and podocyte injury could be summarized as follows: both hyperglycemia and AGE could initiate endoplasmic reticulum stress and activate mTORC-1 protein. Furthermore, continuous injury will contribute to podocyte apoptosis by the caspase-12 pathway, while AGE could act on collagen type IV, leading to podocytes loss or dysfunction $[15,72]$.

4.4. mTOR Signaling Pathways Mediated by Autophagy in $D N$. Studies have shown that intervention in the activity of the mTOR signaling pathway is likely to aggravate podocyte injury in DN renal tissue [73]. Activated podocytes in mTORC1 could result in dislocation of nephrin protein, ZO-1 (skeleton protein) disorders, and podocyte EMT, which could eventually lead to podocyte detachment, foot process fusion and disappearance, and other podocyte injuries in Inoki knockout PcKO Tscl mouse. In conclusion, mTORC1 activity has a key regulatory role in podocyte injury on the DN model of rats [15]. In addition, many pharmacological studies of mTOR inhibitors have further elucidated the importance of mTOR in mediating DN podocyte injury from another perspective such as rapamycinprotected podocytes $[15,74]$. Recent researches have indicated that autophagy, a protective mechanism of podocyte, was used to against damage in a variety of pathological factors. On the contrary, autophagy defects not only led to podocyte injury and proteinuria but also aggravated glomerular sclerosis $[54,75,76]$. Rapamycin, as the mTOR inhibitor, acted on the autophagy pathway and protected the podocyte [55]. In conclusion, there were two kinds of defense mechanism that the autophagy/mTOR signal pathway is using to protect podocytes from further injury. The pathway of mTORC1 is activated and the protective autophagy is inhibited when podocyte is in a high-glucose environment. Specifically, mTORC1 inhibited the autophagosome by initiating the activity of UNC-51-like kinase 1 (ULK1) [13, 77]. In addition, rapamycin could upregulate the protein expression of 1A/1B light chain 3 (LC3) in vitro and improved podocyte autophagy disorders in sequence [78].

4.5. Rho/ROCK Signaling Pathway in DN. The Rho family is mainly composed of RhoA, Rac1, and Cdc42. They were the vital mediators of the actin cytoskeleton protein structure [79]. RhoA/ROCK pathway was the important process in the progression of DN and could induce downstream signaling element cell apoptosis, migration, and differentiation [80]. Immoderate activities of Racl, a key element in the Rho GTPases family, could cause macroalbuminuria quickly with focal foot process effacement, indicating podocyte apoptosis and slit diaphragm protein expression reductions in high glucose [81, 82]. Wang et al. [83] found that Drp1 at serine 600 , as a substrate of Rho signal pathway, not only initiated mitochondrial ROS and podocyte apoptosis in high glucose but also was phosphorylated in ROCK1 knock-out mouse. RhoA played an important role in glomerular filtration barrier integrity, and its overexpression could damage the structure and function of the barrier [84, 85]. Phosphatase and tensin homolog (PTEN) could inhibit the activation of the RhoA/Rac1/Cdc42 signaling pathway, which contributed to reverse the cytoskeletal rebuilding and prevent the development of DN [86, 87]. Previous studies demonstrated that Rho-GTPase family elements were probably activated to induce the downstream cascade reaction when they were exposed in the environment of diabetics, such as AGEs, 


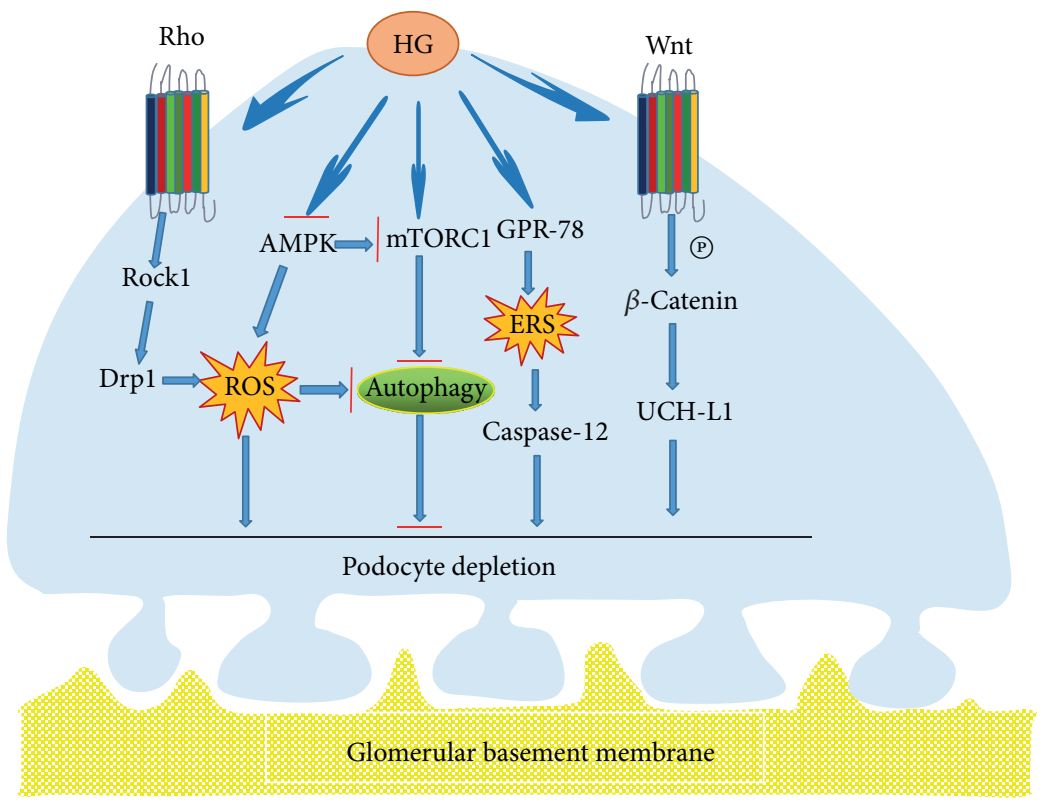

HG High glucose

FIGURE 5: Signaling pathways underlying podocyte injury in DN. Hyperglycemia induced podocyte injury through the Rho/ROCK1 and AMPK signal pathways. Part elements of the signal pathway initiated autophagy that protects from podocyte injury through ROS or mTORC1 participation in the active process. Additionally, GPR-78 activated ERS to induce podocyte apoptosis based on the caspase-12 signal pathway, and the $\mathrm{Wnt} / \beta$-catenin signal pathway may have similar changes in podocyte injury, which could induce podocyte depletion via upregulating expression of UCH-L1 in high glucose.

hyperglycemia, oxidized LDL, and ROS [88, 89]. Xie et al. [90] suggested that Berberine not only inhibited RhoA/ ROCK to improve DN but also regulated Rho GTPases to reduce oxidative stress.

\section{Conclusion}

Podocyte injury is an important factor in DN progression. Several studies implied that the process of albuminuria development in DN was complicated, which presumably included four phases in sequence as follows: podocyte hypertrophy, podocyte EMT, podocyte detachment, and podocyte apoptosis, serving as the warning mark of GFR in DN [91]. More and more signal pathways which induced podocyte injury have been discovered [92], such as $\mathrm{Wnt} / \beta$-catenin signaling pathways, Rho-GTPase signaling pathways, mTOR signaling pathways, and endoplasmic reticulum stress-related signaling pathways (Figure 5). People have understood the multiple pathogenesis mechanisms of podocyte injury in $\mathrm{DN}$, but the complex clinical manifestations of that tell us that there is still potential knowledge required to study and discuss.

\section{Conflicts of Interest}

The authors confirm that there is no conflict of interests.

\section{Acknowledgments}

This work was supported by grants from the National Science and Technology Major Project (no. 81673907) and Beijing Municipal Science \& Technology Project (no. Z161100000516024).

\section{References}

[1] L. Gnudi, R. J. Coward, and D. A. Long, "Diabetic nephropathy: perspective on novel molecular mechanisms," Trends in Endocrinology and Metabolism, vol. 27, no. 11, pp. 820-830, 2016.

[2] K. Drummond, M. Mauer, and International Diabetic Nephropathy Study Group, "The early natural history of nephropathy in type 1 diabetes. II. Early renal structural changes in type 1 diabetes," Diabetes, vol. 51, no. 5, pp. 1580-1587, 2002.

[3] M. E. Pagtalunan, P. L. Miller, S. Jumping-Eagle et al., "Podocyte loss and progressive glomerular injury in type II diabetes," The Journal of Clinical Investigation, vol. 99, no. 2, pp. 342-348, 1997.

[4] M. Dalla Vestra, A. Masiero, A. M. Roiter, A. Saller, G. Crepaldi, and P. Fioretto, "Is podocyte injury relevant in diabetic nephropathy? Studies in patients with type 2 diabetes," Diabetes, vol. 52, no. 4, pp. 1031-1035, 2003.

[5] Y. Maezawa, M. Takemoto, and K. Yokote, "Cell biology of diabetic nephropathy: roles of endothelial cells, tubulointerstitial cells and podocytes," Journal of Diabetes Investigation, vol. 6, no. 1, pp. 3-15, 2015.

[6] D. B. Johnstone and L. B. Holzman, "Clinical impact of research on the podocyte slit diaphragm," Nature Clinical Practice Nephrology, vol. 2, no. 5, pp. 271-282, 2006.

[7] T. Takeda, "Podocyte cytoskeleton is connected to the integral membrane protein podocalyxin through $\mathrm{Na}+/ \mathrm{H}+$-exchanger 
regulatory factor 2 and ezrin," Clinical and Experimental Nephrology, vol. 7, no. 4, pp. 260-269, 2003.

[8] T. Imasawa, E. Obre, N. Bellance et al., "High glucose repatterns human podocyte energy metabolism during differentiation and diabetic nephropathy," The FASEB Journal, vol. 31, no. 1, pp. 294-307, 2016.

[9] J. S. Lin and K. Susztak, "Podocytes: the weakest link in diabetic kidney disease," Current Diabetes Reports, vol. 16, no. 5, p. 45, 2016.

[10] N. Herbach, I. Schairer, A. Blutke et al., "Diabetic kidney lesions of GIPRdn transgenic mice: podocyte hypertrophy and thickening of the GBM precede glomerular hypertrophy and glomerulosclerosis," American Journal of Physiology - Renal Physiology, vol. 296, no. 4, pp. 819-829, 2009.

[11] N. H. Kim, "Podocyte hypertrophy in diabetic nephropathy," Nephrology (Carlton), vol. 10, Supplement, pp. S14-S16, 2005.

[12] M. Romero, A. Ortega, A. Izquierdo, P. López-Luna, and R. J. Bosch, "Parathyroid hormone-related protein induces hypertrophy in podocytes via TGF- $\beta 1$ and $27 \mathrm{Kip} 1$ : implications for diabetic nephropathy," Nephrology, Dialysis, Transplantation, vol. 25, no. 8, pp. 2447-2457, 2010.

[13] M. K. Lu, X. G. Gong, and K. L. Guan, "mTOR in podocyte function: is rapamycin good for diabetic nephropathy?," Cell Cycle, vol. 10, no. 20, pp. 3415-3416, 2011.

[14] M. Gödel, B. Hartleben, N. Herbach et al., "Role of mTOR in podocyte function and diabetic nephropathy in humans and mice," The Journal of Clinical Investigation, vol. 121, no. 6, pp. 2197-2209, 2011.

[15] K. Inoki, H. Mori, J. Wang et al., "mTORC1 activation in podocytes is a critical step in the development of diabetic nephropathy in mice," The Journal of Clinical Investigation, vol. 121, no. 6, pp. 2181-2196, 2011.

[16] H. A. Jo, J. Y. Kim, S. H. Yang et al., “The role of local IL6/ JAK2/STAT3 signaling in high glucose-induced podocyte hypertrophy," Kidney Research Clinical Practice, vol. 35, no. 4, pp. 212-218, 2016.

[17] D. K. Kim, B. Y. Nam, J. J. Li et al., "Translationally controlled tumour protein is associated with podocyte hypertrophy in a mouse model of type 1 diabetes," Diabetologia, vol. 55, no. 4 , pp. 1205-1217, 2012.

[18] N. H. Kim, H. Rincon-Choles, B. Bhandari, G. G. Choudhury, H. E. Abboud, and Y. Gorin, "Redox dependence of glomerular epithelial cell hypertrophy in response to glucose," American Journal of Physiology - Renal Physiology, vol. 290, no. 3, pp. 741-751, 2006.

[19] Y. Liu, "Epithelial to mesenchymal transition in renal fibrogenesis: pathologic significance molecular mechanism, and therapeutic intervention," Journal of the American Society Nephrology, vol. 15, no. 1, pp. 1-12, 2004.

[20] J. P. Thiery and J. P. Sleeman, "Complex networks orchestrate epithelial-mesenchymal transitions," Nature Reviews Molecular Cell Biology, vol. 7, no. 2, pp. 131-142, 2006.

[21] Q. Lu, X. J. Ji, Y. X. Zhou et al., "Quercetin inhibits the mtorc1/p70s6k signaling-mediated renal tubular epithelialmesenchymal transition and renal fibrosis in diabetic nephropathy," Pharmacological Research, vol. 99, pp. 237247, 2015.

[22] I. Loeffler and G. Wolf, "Epithelial-to-mesenchymal transition in diabetic nephropathy: fact or fiction?," Cell, vol. 4, no. 4, pp. 631-652, 2015.
[23] L. Liu, W. Fu, J. Xu, L. Shao, and Y. Wang, "Effect of bmp7 on podocyte transdifferentiation and smad7 expression induced by hyperglycemia," Clinical Nephrology, vol. 84, no. 2, pp. 95-99, 2015.

[24] H. Y. Dai, M. Zheng, R. N. Tang et al., "Effects of angiotensin receptor blocker on phenotypic alterations of podocytes in early diabetic nephropathy," The American Journal of the Medical Sciences, vol. 341, no. 3, pp. 207-214, 2011.

[25] Y. Yamaguchi, M. Iwano, D. Suzuki et al., "Epithelialmesenchymal transition as a potential explanation for podocyte depletion in diabetic nephropathy," American Journal Kidney Diseases, vol. 54, no. 4, pp. 653-664, 2009.

[26] L. Xing, Q. Liu, S. Fu et al., "PTEN inhibits high glucose-induced phenotypic transition in podocytes," Journal of Cellular Biochemistry, vol. 116, no. 8, pp. 1776-1784, 2015.

[27] Y. Li, Y. S. Kang, C. Dai, L. P. Kiss, X. Wen, and Y. Liu, "Epithelial-to-mesenchymal transition is a potential pathway leading to podocyte dysfunction and proteinuria," The American Journal of Pathology, vol. 172, no. 2, pp. 299-308, 2008.

[28] S. U. Vogelmann, W. J. Nelson, B. D. Myers, and K. V. Lemley, "Urinary excretion of viable podocytes in health and renal disease," American Journal of Physiology - Renal Physiology, vol. 285, no. 1, pp. F40-F48, 2003.

[29] D. Yu, A. Petermann, U. Kunter, S. Rong, S. J. Shankland, and J. Floege, "Urinary podocyte loss is a more specific marker of ongoing glomerular damage than proteinuria," Journal of the American Society of Nephrology, vol. 16, no. 6, pp. 17331741, 2005.

[30] S. Mathew, X. Chen, A. Pozzi, and R. Zent, "Integrins in renal development," Pediatric Nephrology, vol. 27, no. 6, pp. 891900, 2012.

[31] B. Jim, M. Ghanta, A. Qipo et al., "Dysregulated nephrin in diabetic nephropathy of type 2 diabetes: a cross sectional study," PLoS One, vol. 7, no. 5, article e36041, 2012.

[32] J. Chen, D. Gui, Y. Chen, L. Mou, Y. Liu, and J. Huang, "Astragaloside IV improves high glucose-induced podocyte adhesion dysfunction via alpha3betal integrin upregulation and integrin-linked kinase inhibition," Biochemical Pharmacology, vol. 76, no. 6, pp. 796-804, 2008.

[33] H. C. Chen, C. A. Chen, J. Y. Guh, J. M. Chang, S. J. Shin, and Y. H. Lai, "Altering expression of alpha3betal integrin on podocytes of human and rats with diabetes," Life Sciences, vol. 67, no. 19, pp. 2345-2353, 2000.

[34] M. Regoli and M. Bendayan, "Alterations in the expression of the alpha 3 beta 1 integrin in certain membrane domains of the glomerular epithelial cells (podocytes) in diabetes mellitus," Diabetologia, vol. 40, no. 1, pp. 15-22, 1997.

[35] M. Miyauchi, M. Toyoda, K. Kobayashi et al., "Hypertrophy and loss of podocytes in diabetic nephropathy," Internal Medicine, vol. 48, no. 18, pp. 1615-1620, 2009.

[36] W. Kriz, B. Hähnel, H. Hosser, S. Rösener, and R. Waldherr, "Structural analysis of how podocytes detach from the glomerular basement membrane under hypertrophic stress," Front Endocrinol (Lausanne), vol. 5, p. 207, 2014.

[37] K. Susztak, A. C. Raff, M. Schiffer, and E. P. Böttinger, "Glucose-induced reactive oxygen species cause apoptosis of podocytes and podocyte depletion at the onset of diabetic nephropathy," Diabetes, vol. 55, no. 1, pp. 225233, 2006. 
[38] P. Y. Chuang, Q. Yu, W. Fang, J. Uribarri, and J. C. He, "Advanced glycation endproducts induce podocyte apoptosis by activation of the FOXO4 transcription factor," Kidney International, vol. 72, no. 8, pp. 965-976, 2007.

[39] A. A. Eid, Y. Gorin, B. M. Fagg et al., "Mechanisms of podocyte injury in diabetes: role of cytochrome P450 and NADPH oxidases," Diabetes, vol. 58, no. 5, pp. 1201-1211, 2009.

[40] T. Niranjan, B. Bielesz, A. Gruenwald et al., "The Notch pathway in podocytes plays a role in the development of glomerular disease," Nature Medicine, vol. 14, no. 3, pp. 290-298, 2008.

[41] R. Bonegio and K. Susztak, "Notch signaling in diabetic nephropathy," Experimental Cell Research, vol. 318, no. 9, pp. 986-992, 2012.

[42] F. Gao, M. Yao, Y. Cao, S. Liu, Q. Liu, and H. Duan, "Valsartan ameliorates podocyte loss in diabetic mice through the Notch pathway," International Journal of Molecular Medicine, vol. 37, no. 5, pp. 1328-1336, 2016.

[43] T. Tejada, P. Catanuto, A. Ijaz et al., "Failure to phosphorylate AKT in podocytes from mice with early diabetic nephropathy promotes cell death," Kidney International, vol. 73, no. 12, pp. 1385-1393, 2008.

[44] W. Liu, Y. Zhang, J. Hao et al., "Nestin protects mouse podocytes against high glucose-induced apoptosis by a Cdk5dependent mechanism," Journal of Cellular Biochemistry, vol. 113, no. 10, pp. 3186-3196, 2012.

[45] J. H. Li, X. R. Huang, H. J. Zhu et al., “Advanced glycation end products activate Smad signaling via TGF-beta-dependent and independent mechanisms: implications for diabetic renal and vascular disease," The FASEB Journal, vol. 18, no. 1, pp. 176178, 2004.

[46] W. T. Liu, F. F. Peng, H. Y. Li et al., "Metadherin facilitates podocyte apoptosis in diabetic nephropathy," Cell Death \& Disease, vol. 7, no. 11, article e2477, 2016.

[47] X. M. Yao, Y. J. Liu, Y. M. Wang, H. Wang, and B. B. Zhu, "Astragaloside IV prevents high glucose-induced podocyte apoptosis via downregulation of TRPC6," Molecular Medicine Reports, vol. 13, no. 6, pp. 5149-5156, 2016.

[48] D. Gui, J. Huang, Y. Guo et al., "Astragaloside IV ameliorates renal injury in streptozotocin-induced diabetic rats through inhibiting NF- $\kappa \mathrm{B}$-mediated inflammatory genes expression," Cytokine, vol. 61, no. 3, pp. 970-977, 2013.

[49] D. Kawanami, K. Matoba, and K. Utsunomiya, "Signaling pathways in diabetic nephropathy," Histology and Histopathology, vol. 31, no. 10, pp. 1059-1067, 2016.

[50] Y. Kim and C. W. Park, "Adenosine monophosphate-activated protein kinase in diabetic nephropathy kidney," Kidney Research and Clinical Practice, vol. 35, no. 2, pp. 69-77, 2016.

[51] S. Kume, M. C. Thomas, and D. Koya, "Nutrient sensing, autophagy, and diabetic nephropathy," Diabetes, vol. 61, no. 1, pp. 23-29, 2012.

[52] S. Alers, A. S. Lffler, S. Wesselborg, and B. Stork, "Role of AMPK-mTOR-UIk1/2 in the regulation of autophagy: cross talk, shortcuts and feedbacks," Molecular and Cellular Biology, vol. 32, no. 1, pp. 2-11, 2012.

[53] J. Kim, M. Kundu, B. Viollet, and K. L. Guan, “AMPK and mTOR regulate autophagy through direct phosphorylation of UIk1," Nature Cell Biology, vol. 13, no. 2, pp. 132-141, 2011.

[54] Y. Tanaka, S. Kume, M. Kitada et al., "Autophagy as a therapeutic target in diabetic nephropathy," Experimental Diabetes Research, vol. 2012, Article ID 628978, 12 pages, 2012.
[55] K. Sharma, S. RamachandraRao, G. Qiu et al., "Adiponectin regulates albuminuria and podocyte function in mice," The Journal of Clinical Investigation, vol. 118, no. 5, pp. 1645-1656, 2008.

[56] H. Levers and R. Nusse, "Wnt/ $\beta$-catenin signaling and disease," Cell, vol. 149, no. 6, pp. 1192-1205, 2012.

[57] I. Matsui, T. Ito, H. Kurihara, E. Imai, T. Ogihara, and H. M. Snail, "Snail, a transcriptional regulator, represses nephrin expression in glomerular epithelial cells of nephrotic rats," Laboratory Investigation, vol. 87, no. 3, pp. 273-283, 2007.

[58] L. Xiao, M. Wang, S. Yang, F. Liu, and L. Sun, "A glimpse of the pathogenetic mechanisms of $\mathrm{Wnt} / \beta$-catenin signaling in diabetic nephropathy," BioMed Research International, vol. 2013, Article ID 987064, 7 pages, 2013.

[59] H. Kato, A. Gruenwald, J. H. Suh et al., "Wnt/ $\beta$-catenin pathway in podocytes integrates cell adhesion, differentiation, and survival," The Journal of Biological Chemistry, vol. 286, no. 29, pp. 26003-26015, 2011.

[60] W. He, Y. S. Kang, C. Dai, and Y. Liu, "Blockade of Wnt/ $\beta$ catenin signaling by paricalcitol ameliorates proteinuria and kidney injury," Journal of American Society of Nephrology, vol. 22, no. 1, pp. 90-103, 2011.

[61] B. L. Liu, Y. P. Chen, H. Cheng et al., "The protective effects of curcumin on obesity-related glomerulopathy are associated with inhibition of $\mathrm{wnt} / \beta$-catenin signaling activation in podocytes," Evidence-Based Complementary and Alternative Medicine, vol. 2015, Article ID 827472, 12 pages, 2015.

[62] H. Zhang, X. Mao, Y. Sun et al., "NF- $\kappa$ B upregulates ubiquitin C-terminal hydrolase 1 in diseased podocytes in glomerulonephritis," Molecular Medicine Reports, vol. 12, no. 2, pp. 2893 2901, 2015.

[63] H. Zhang, Y. Sun, R. Hu et al., "The regulation of the UCH-L1 gene by transcription factor NF- $\kappa$ B in podocytes," Cellular Signalling, vol. 25, no. 7, pp. 1574-1585, 2013.

[64] H. Zhang, W. Luo, Y. Sun, Y. Qiao, and L. Zhang, "Wnt/ $\beta$ catenin signaling mediated-UCH-L1 expression in podocytes of diabetic nephropathy," International Journal of Molecular Sciences, vol. 17, no. 9, article E1404, 2016.

[65] Z. Li, J. Xu, P. Xu, S. Liu, and Z. Yang, "Wnt/ $\beta$-catenin signalling pathway mediates high glucose induced cell injury through activation of TRPC6 in podocytes," Cell Proliferation, vol. 46, no. 1, pp. 76-85, 2013.

[66] Y. Yuan, X. Xu, C. Zhao et al., "The roles of oxidative stress, endoplasmic reticulum stress, and autophagy in aldosterone mineralocorticoid receptor-induced podocyte injury," Laboratory Investigation, vol. 95, no. 12, pp. 1374-1386, 2015.

[67] G. Rong, X. Tang, T. Guo et al., “Advanced oxidation protein products induce apoptosis in podocytes through induction of endoplasmic reticulum stress," Journal of Physiology and Biochemistry, vol. 71, no. 3, pp. 455-470, 2015.

[68] Y. Cao, Y. Hao, H. Li et al., "Role of endoplasmic reticulum stress in apoptosis of differentiated mouse podocytes induced by high glucose," International Journal of Molecular Medicine, vol. 33, no. 4, pp. 809-816, 2014.

[69] Y. Chen, C. P. Liu, K. F. Xu et al., "Effect of taurine-conjugated ursodeoxycholic acid on endoplasmic reticulum stress and apoptosis induced by advanced glycation end products in cultured mouse podocytes," American Journal of Nephrology, vol. 28, no. 6, pp. 1014-1022, 2008.

[70] S. M. Colgan, A. A. Hashimi, and R. C. Austin, "Endoplasmic reticulum stress and lipid dysregulation," Expert Reviews in Molecular Medicine, vol. 13, article e4, 2011. 
[71] A. Zhuang and J. M. Forbes, "Stress in the kidney is the road to pERdition: is endoplasmic reticulum stress a pathogenic mediator of diabetic nephropathy?," The Journal of Endocrinology, vol. 222, no. 3, pp. R97-R111, 2014.

[72] M. Sakaguchi, M. Isono, K. Isshiki, T. Sugimoto, D. Koya, and A. Kashiwagi, "Inhibition of mTOR signaling with rapamycin attenuates renal hypertrophy in the early diabetic mice," Biochemical and Biophysical Research Communications, vol. 340, no. 1, pp. 296-301, 2006.

[73] M. Pourghasem, H. Shafi, and Z. Babazadeh, "Histological changes of kidney in diabetic nephropathy," Caspian Journal of Internal Medicine, vol. 6, no. 3, pp. 120-127, 2015.

[74] L. Wu, Z. Feng, S. Cui et al., "Rapamycin upregulates autophagy by inhibiting the mTOR-ULK1 pathway, resulting in reduced podocyte injury," PLoS One, vol. 8, no. 5, article e63799, 2013.

[75] B. Hartleben, M. Gödel, C. Meyer-Schwesinger et al., “Autophagy influences glomerular disease susceptibility and maintains podocyte homeostasis in aging mice," The Journal of Clinical Investigation, vol. 120, no. 4, pp. 1084-1096, 2010.

[76] Y. Ding and M. E. Choi, "Autophagy in diabetic nephropathy," The Journal of Endocrinology, vol. 224, no. 1, pp. R15-R30, 2015.

[77] N. Hosokawa, T. Hara, T. Kaizuka et al., "Nutrient-dependent mTORC1 association with the ULK1-Atg13-FIP200 complex required for autophagy," Molecular Biology of the Cell, vol. 20, no. 7, pp. 1981-1991, 2009.

[78] T. Xiao, X. Guan, L. Nie et al., "Rapamycin promotes podocyte autophagy and ameliorates renal injury in diabetic mice," Molecular and Cellular Biochemistry, vol. 394, no. 1-2, pp. 145-154, 2014.

[79] S. M. Blattner, J. B. Hodgin, M. Nishio et al., "Divergent functions of the Rho GTPases Racl and Cdc42 in podocyte injury," Kidney International, vol. 84, no. 5, pp. 920-930, 2013.

[80] R. Komers, "Rho kinase inhibition in diabetic kidney disease," British Journal of Clinical Pharmacology, vol. 76, no. 4, pp. 551-559, 2013.

[81] H. Yu, H. Suleiman, A. H. Kim et al., "Rac1 activation in podocytes induces rapid foot process effacement and proteinuria," Molecular and Cellular Biology, vol. 33, no. 23, pp. 47554764, 2013.

[82] M. Ishizaka, T. Gohda, M. Takagi et al., "Podocyte-specific deletion of Rac1 leads to aggravation of renal injury in STZinduced diabetic mice," Biochemical and Biophysical Research Communications, vol. 467, no. 3, pp. 549-555, 2015.

[83] W. Wang, Y. Wang, J. Long et al., "Mitochondrial fission triggered by hyperglycemia is mediated by rock1 activation in podocytes and endothelial cells," Cell Metabolism, vol. 15, no. 2, pp. 186-200, 2012.

[84] K. Asanuma, E. Yanagida-Asanuma, C. Faul, Y. Tomino, K. Kim, and P. Mundel, "Synaptopodin orchestrates actin organization and cell motility via regulation of RhoA signalling," Nature Cell Biology, vol. 8, no. 5, pp. 485-491, 2006.

[85] L. Wang, M. J. Ellis, J. A. Gomez, W. Eisner, and W. Fennell, "Mechanisms of the proteinuria induced by Rho GTPases," Kidney International, vol. 81, no. 11, pp. 1075-1085, 2012.

[86] J. S. Lin, Y. Shi, H. Peng et al., "Loss of PTEN promotes podocyte cytoskeletal rearrangement, aggravating diabetic nephropathy," The Journal of Pathology, vol. 236, no. 1, pp. 30-40, 2015.
[87] H. Y. Gee, P. Saisawat, S. Ashraf, T. W. Hurd, and V. VegaWarner, "ARHGDIA mutations cause nephrotic syndrome via defective RHO GTPase signaling," The Journal of Clinical Investigation, vol. 123, no. 8, pp. 3243-3253, 2013.

[88] F. Peng, D. Wu, B. Gao, A. J. Ingram, and B. Zhang, "RhoA/ Rho-kinase contribute to the pathogenesis of diabetic renal disease," Diabetes, vol. 57, no. 6, pp. 1683-1692, 2008.

[89] G. I. Welsh, L. J. Hale, V. Eremina, M. Jeansson, and Y. Maezawa, "Insulin signaling to the glomerular podocyte is critical for normal kidney function," Cell Metabolism, vol. 12, no. 4, pp. 329-340, 2010.

[90] X. Xie, X. Chang, L. Chen, K. Huang, and J. Huang, "Berberine ameliorates experimental diabetes-induced renal inflammation and fibronectin by inhibiting the activation of RhoA/ ROCK signaling," Molecular and Cellular Endocrinology, vol. 381, no. 1-2, pp. 56-65, 2013.

[91] S. S. Guhr, M. Sachs, A. Wegner, J. U. Becker, and T. N. Meyer, "The expression of podocyte-specific proteins in parietal epithelial cells is regulated by protein degradation," Kidney International, vol. 84, no. 3, pp. 532-544, 2013.

[92] F. C. Brosius, C. C. Khoury, C. L. Buller, and S. Chen, "Abnormalities in signaling pathways in diabetic nephropathy," Expert Review of Endocrinology and Metabolism, vol. 5, no. 1, pp. 51-64, 2010. 


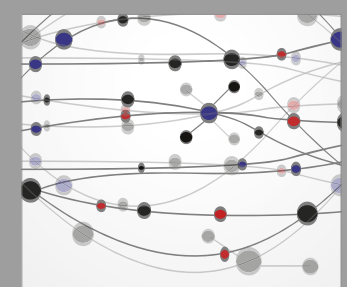

The Scientific World Journal
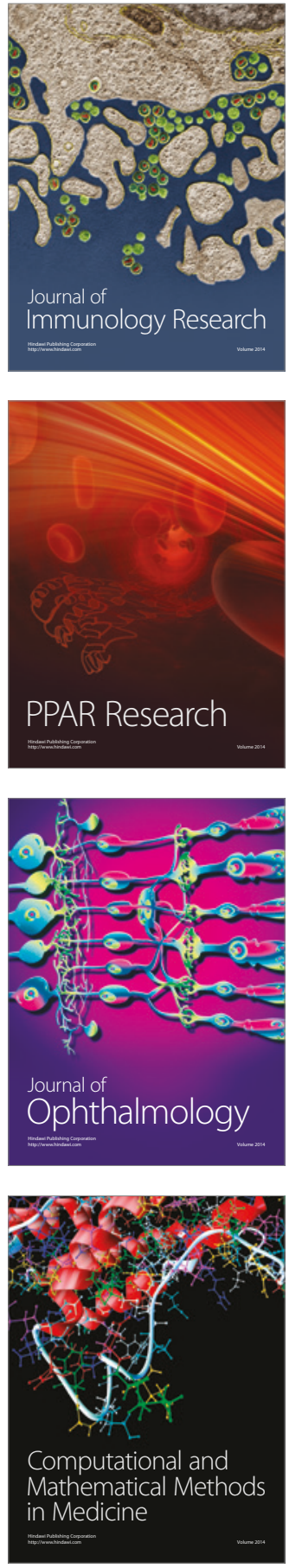

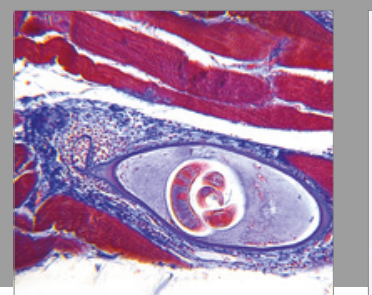

Gastroenterology Research and Practice
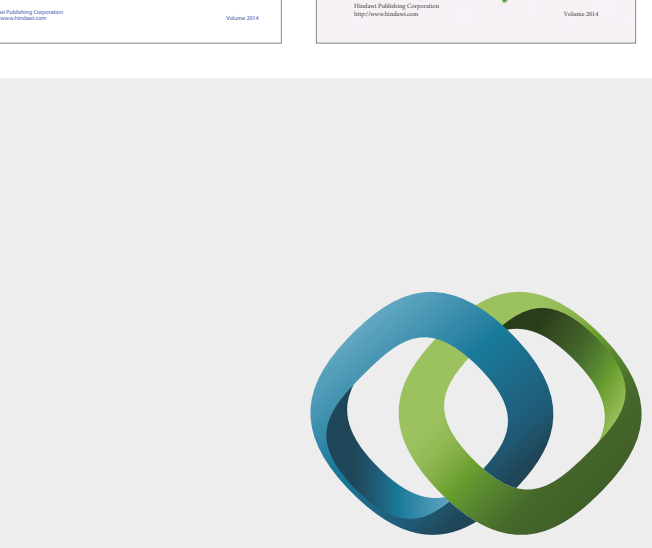

\section{Hindawi}

Submit your manuscripts at

https://www.hindawi.com
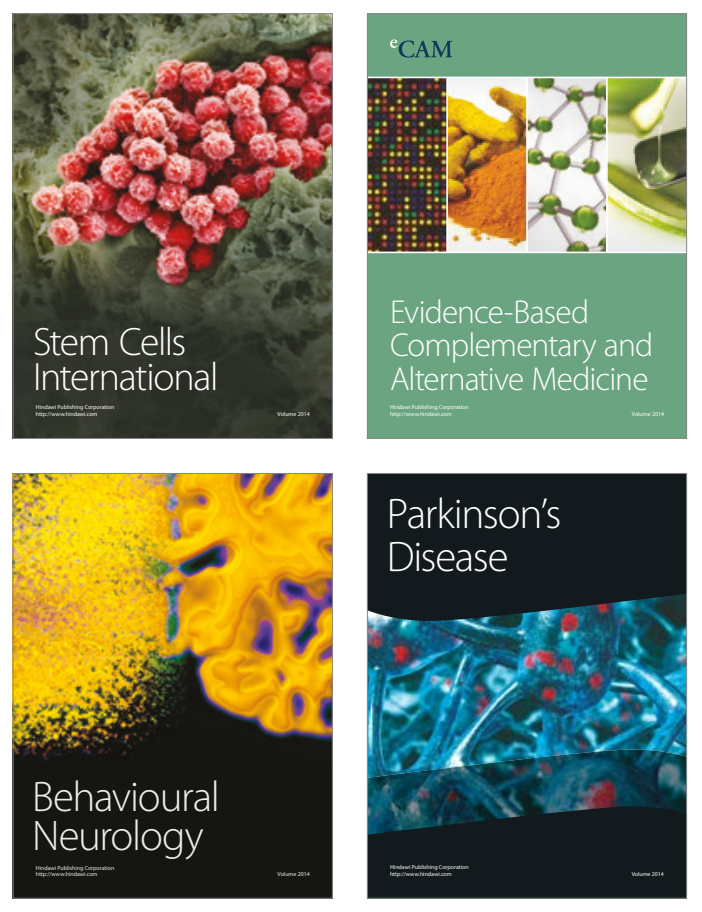
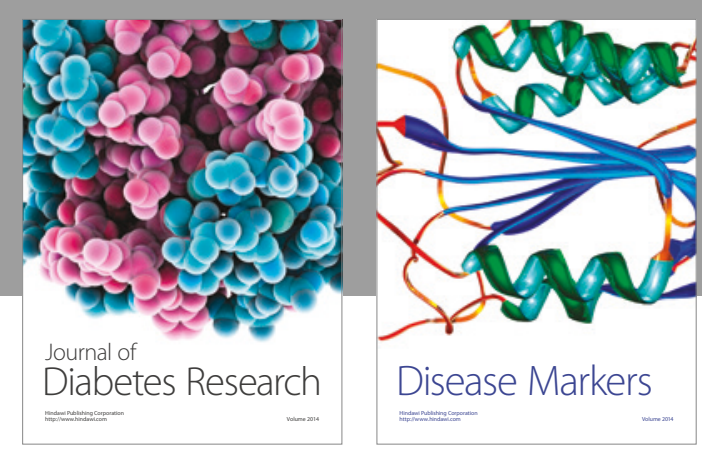

Disease Markers
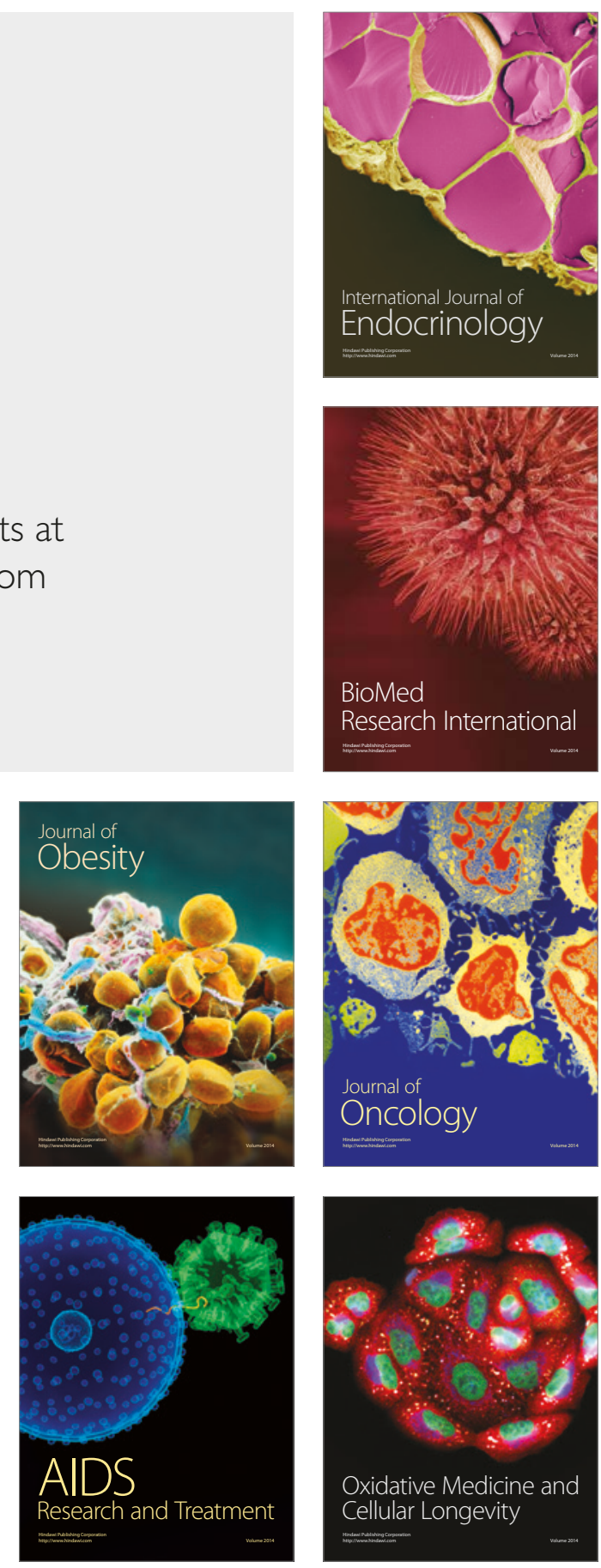